\title{
Review
}

\section{Vesicular trafficking and autophagosome formation}

\author{
A Longatti ${ }^{1}$ and SA Tooze ${ }^{*, 1}$
}

The source of the autophagosome membrane, and the formation of the autophagosome remain the most important questions for understanding autophagy. Fundamentally, the process of autophagosome formation is similar between yeast and mammalian cells and many of the proteins involved (called the autophagy-related (Atg) proteins) are known, having been first discovered in yeast. However, both in yeast and mammalian cells, the molecular details are missing to explain how the double-membrane autophagosome is formed. Important advances in our understanding of the formation process have recently been obtained, and here, we review and interpret these data in the context of well-known paradigms of membrane trafficking to develop some hypothetical models for how an autophagosome forms in mammalian cells.

Cell Death and Differentiation (2009) 16, 956-965; doi:10.1038/cdd.2009.39; published online 17 April 2009

In mammalian cells, autophagy is an inducible cell survival pathway that relies on the lysosomal degradation pathway for its execution (Figure 1). Autophagosomes sequester cytosolic components constitutively, or during the times of nutrient deprivation or stress, and fuse with the endosomal and lysosomal system to acquire degradative enzymes to digest the sequestered material. ${ }^{1}$ In mammalian cells, autophagy is initiated by the formation or elongation of the isolation membrane (IM), also called a phagophore. In yeast, the IM arises from the yeast pre-autophagosomal structure (PAS), ${ }^{2}$ the PAS is defined as the initial site of autophagy-related (Atg) protein recruitment. In mammalian cells, so far there is no evidence for a PAS, and so the mammalian IM could either be derived from the equivalent of the yeast PAS, or be, in fact, the PAS itself. One of the major differences between yeast and mammalian autophagy seems to be that, in yeast, all autophagosomes apparently arise from a single PAS, whereas in mammalian cells, it appears that they can be generated anywhere in the cytoplasm. The IM is induced to grow and expand into a double-membrane vesicle (Figure 1). Expansion of the IM requires a unique set of proteins and is followed by the closure of the double membrane forming a spherical immature autophagosome. The full molecular details of how the IM expands and closes remain to be understood. Autophagy is completed by fusion of autophagic vacuole (AV)i (immature autophagosomes) with endosomes and lysosomes, forming an AVd (degradative autophagosome) or autolysosome, followed by consumption of the content, and release of amino acids and other building blocks into the cytosol.
Much of the molecular details about the autophagosome formation were originally obtained in the yeast. Among the 31 Atg proteins in yeast, 18 have been shown to be involved in autophagosome formation (Atg1-10, Atg12-14, Atg16-18, Atg29, and Atg31). ${ }^{3,4}$ Most, if not all, of these proteins are recruited to the PAS, on which some remain throughout the autophagosome maturation and can be used as markers, in particular, Atg8 (or microtubule-associated protein light chain 3 (LC3) in mammals). Genetic and biochemical studies have elucidated many of the interactions between these proteins as well as the sequence of their action, allowing a hierarchy to be established. However, this hierarchy reveals a complex interrelationship of the Atg proteins and the molecular events they mediate, which do not occur in a linear sequence. All the data to date suggest that the hierarchy in yeast and mammalian cells are identical or very similar. Owing to this complex hierarchy, the most 'upstream' event, the initiating event in autophagosome formation, has been so far impossible to identify. The identification of this initiating event combined with an increased understanding of what the IM/PAS is, the sequence of recruitment of Atg proteins to IMs, and the source of components allowing expansion could finally define the mysterious membrane source of the autophagosome.

\section{Molecular Machinery Required for Induction of Autophagy}

Recent data have dramatically expanded our knowledge of how the Atg proteins function to expand the IM into an autophagosome. This has mostly come about through work

\footnotetext{
${ }^{1}$ Cancer Research UK, London Research Institute, Secretory Pathways Laboratory, 44 Lincolns Inn Fields, London WC2A 3PX, UK

${ }^{*}$ Correspondence: S Tooze, Secretory Pathways Laboratory, Cancer Research UK, London Research Institute, 44 Lincolns Inn Fields, London WC2A 3PX, UK. Tel: + 44207269 3122; Fax: + 44207269 3417; E-mail: sharon.tooze@ cancer.org.uk

Keywords: autophagy; autophagosome; isolation membrane; phagophore; membrane trafficking; Atg proteins

Abbreviations: Atg, autophagy-related; AV, autophagic vacuole; AVi, immature AV; AVd, degradative AV; BIF-1, Bax-interacting factor 1; DFCP1, Double FYVE domain-containing protein 1; ERGIC, ER-Golgi intermediate compartment; ESCRT, endosomal sorting complex required for transport; FIP200, focal adhesion kinase family interacting protein of $200 \mathrm{kD}$; IM, isolation membrane; LC3, microtubule-associated protein light chain 3; PAS, pre-autophagosomal structure; PE, phosphatidylethanolamine; PI3P, phophatidylinositol-3-phosphate; SNARE, soluble $N$-ethylmaleimide-sensitive factor attachment protein receptors; TGN, trans-Golgi network; TOR, target of rapamycin; TP53INP2, tumour protein 53 induced nuclear protein 2; ULK, unc-51-like kinase; UVRAG, ultraviolet irradiation resistant-associated gene; VMP1, vacuolar membrane protein 1; Vps, vacuolar protein sorting; WIPI, WD-repeat protein interacting with phosphoinositides Received 22.12.08; revised 02.3.09; accepted 12.3.09; Edited by M Piacentini; published online 17.4.09
} 


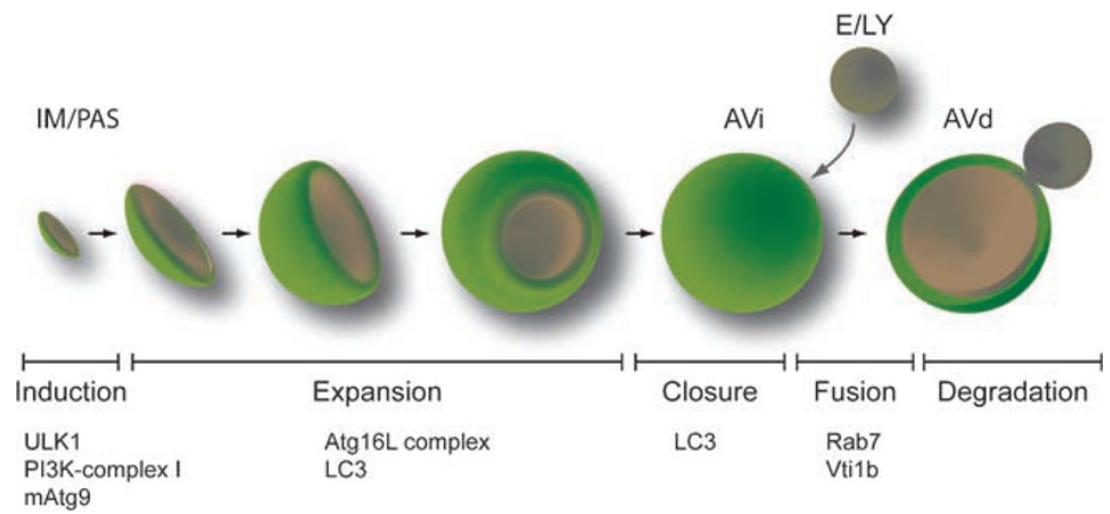

Figure 1 Scheme of macroautophagy pathway in mammalian cells. Autophagy is initiated during an induction phase on IMs/PAS (isolation membranes/preautophagosomal structures) that serves both as a signalling platform and a membrane source/acceptor compartment (see Figure 2 for IM-associated molecules). The source of the IM is unknown. During the expansion phase, the IM grows sequestering cytosolic components (for simplicity the sequestered cytosolic components are not illustrated). The possible mechanisms for expansion of the double-membrane IM are addressed in Figure 4. After closure of the expanded IM, the immature autophagosome (AVi) fuses with the endosomal compartments $(E)$ and lysosomes $(L Y)$, becoming a degradative autophagosome AVd. Degradation of the sequestered cytosolic components occurs in the $\mathrm{AVd}$ as it matures into an autolysosome. At the final stage, the AVd is shown in cross-section to aid visualization of the membrane fusion event(s)

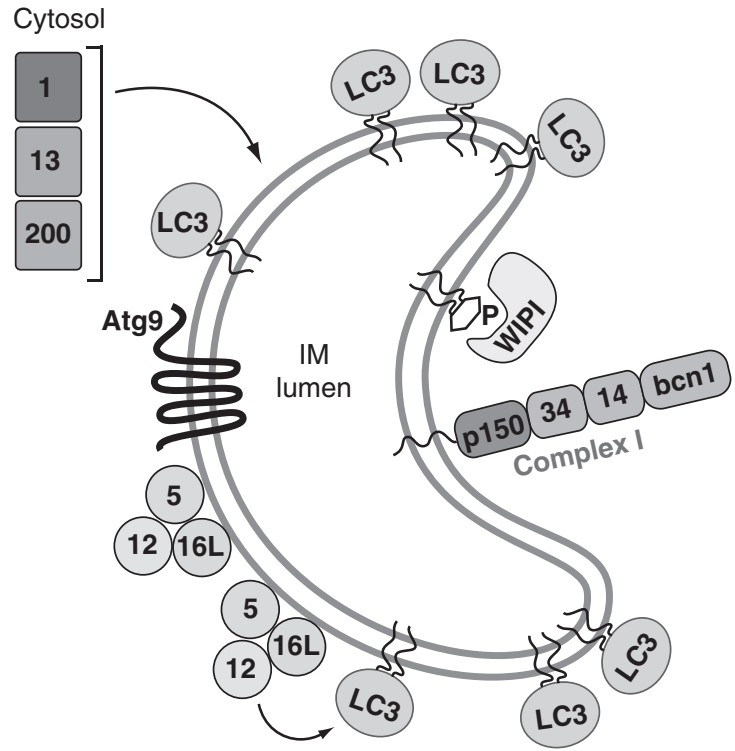

Figure 2 Proteins localized to the isolation membrane (IM). Proteins required for the expansion of the IM are shown on the membrane by their mode of association (if known). For example, the PI3K complex I associates with the membrane through $\mathrm{p} 150$, which has an N-terminal myristoylation acceptor sequence, ${ }^{79} \mathrm{LC} 3$ is bound through $\mathrm{PE}$, and mAtg9 has six transmembrane domains. It is not known how ULK1 or the Atg16L complexes are associated with the membrane. 1, ULK1; 5, Atg5; 12, Atg12; 13, Atg13; 16L, Atg16L; 200, FIP200; 34 , Vps 34; 14, Atg14; bcn1, Beclin1

on (1) the two ubiquitin-related pathways (the Atg5-12 and Atg8-PE (Atg8-phosphatidylethanolamine) conjugation systems); (2) the regulation of the class III phosphatidylinositol3-phosphate (PI3P) kinase complex I (PI3K complex I), which forms PI3P, an essential phospholipid for IM expansion, and its interactors; (3) the transmembrane protein, mammalian Atg (mAtg)9; and (4) the Atg1 kinase homologues, unc-51-like kinase (ULK) 1 and ULK2. As in yeast, these are all present on the IM (Figure 2). We will briefly review advances in the field with emphasis on how our knowledge about these molecules has increased our understanding of the formation and expansion process.

Ubiquitin-like conjugation pathways. In the first ubiquitinrelated system, Atg12 is conjugated to Atg5 by the combined action of Atg7 and Atg10 (E1 and E2-like enzymes, respectively). The Atg5-12 conjugate, the formation of which is essentially constitutive, associates with Atg16L, and this trimeric complex homodimerizes to form a multimeric $800 \mathrm{kDa}$ complex called the Atg16 $\mathrm{L}$ complex. ${ }^{5}$ The second ubiquitin-related system leads to the conjugation of LC3 (the homologue of Atg8 in yeast) to the lipid PE by Atg7 and Atg3, the latter of which acts as an E2-like enzyme in this conjugation reaction. The lipidated form of LC3 is referred to as LC3-II and localizes to autophagosomal membranes, whereas the unlipidated, cytosolic form is called LC3-I. Importantly, recent data suggests that the Atg16L complex associates with the IM by an unknown mode of recognition, possibly involving Rab33b (see below), and here it can act as a novel E3-like enzyme allowing the recruitment of the Atg3 (E2)-LC3 intermediate to the substrate PE in the membrane, thus determining the site of LC3 lipidation. ${ }^{6,7}$

Elongation of the IM requires the Atg16 $\mathrm{L}$ complex $^{8}$ and LC3-II/Atg8-PE. The properties of the Atg16L complex suggest that it may provide a way to determine where the autophagosome membrane expansion starts. Atg8-PE has fusogenic properties, ${ }^{9}$ which could catalyse the elongation process. Recently, it was shown that ectopic localization of Atg16L to the plasma membrane promoted recruitment of LC3-II to this site: Atg16L was targeted to the plasma membrane by the addition of a C-terminal prenylation motif (CAAX) derived from K-Ras. ${ }^{6}$ The Atg16L-CAAX fusion protein, through Atg12, recruited GFP-LC3 to the plasma membrane, where it was lipidated by the Atg3-catalysed transfer to PE. Interestingly, GFP-LC3 lipidation was starvation-independent and insensitive to wortmannin, which inhibits the PI3K complex I, suggesting that the main output of PI3P formation is the localization of the Atg16 L complex to the PI3P-rich IM. In addition, the expression of the Atg16L- 
CAAX-containing complex inhibited the starvation-induced formation of LC3-positive autophagosomes in the cytosol, presumably by competition for the endogenous pools of Atg4, Atg7, and Atg3.

The lipidation of LC3 (and its paralogues GATE-16, GABARAP, and Atg8L) requires prior cleavage by Atg4, a cysteine protease. Importantly, Atg4 can also act on LC3-II to release LC3 from $P E$, and therefore from the autophagosomal membrane. The removal of LC3-II from the autophagosome is required for its subsequent fusion with the endosome/ lysosome. ${ }^{10}$ There are four members of the Atg 4 family, Atg $4 a-d$, also called the autophagins $1-4,{ }^{11}$ and among these Atg4b seems to have the broadest specificity. ${ }^{12-14}$

The Atg4b-dependent cleavage of LC3 requires the catalytic residue, Cys74, and mutation of this residue has been shown to inhibit the cleavage of LC3-II and GABARAP-II in vitro and in vivo in cell culture model systems. Overexpression of Atg4b was also found to inhibit the lipidation of LC3, ${ }^{14}$ and the formation of GFP-LC3-positive autophagosomes. ${ }^{15}$ The expression of the catalytically inactive mutant, Atg4b-C47A, however, provided a more interesting phenotype. Although there was no effect on the Atg16L complex formation or its recruitment to autophagosomes, the lifetime of the Atg5-12 conjugate on membranes increased from $5 \mathrm{~min}$ to $20 \mathrm{~min}$ in cells overexpressing Atg4b-C47A. In these overexpressing cells, autophagosomes were readily detected and a larger percentage of open IMs were found. Although the length of the IMs was not altered after Atg4b-C74A expression, the closed autophagosomes were smaller than those seen in control cells. mAtg9 localization was not affected and ULK1 colocalization with Atg5 was also not perturbed. ${ }^{15}$ Note, ULK1 is also recruited to Atg5-positive autophagosomes formed in cells expressing conjugation-defective Atg5-K130R mutant, which has mini-IMs. ${ }^{8}$

Lastly, in mouse embryonic fibroblasts (MEFs) isolated from $\mathrm{Atg}^{-1-}$ mice several important observations were made about the inter-relationship between the Atg5-12 and LC3/Atg8 conjugation systems, and the role of these systems in autophagy. In the $A \operatorname{tg} 3^{-1-}$ MEFs, there was a reduced conjugation of Atg12 to Atg5, and no LC3-II, GABARAP-II, or GATE16-II could be detected. However, under starvation conditions, autophagosomes were detected by EM analysis, but they were smaller than in wild-type (wt) cells. Atg5 and Atg16L were recruited to and remained longer on IMs compared with IMs in wt MEFs. Interestingly, in $\mathrm{Atg}^{-/-}$cells, IMs were reported to be abnormally shaped, curling around multiple sections of cytosol, and appeared either open ended or multi-lamellar. ${ }^{16}$

These data provide evidence that the elongation and closure of the IM is controlled by the Atg16 complex and by the LC3-lipidation system. The intimate inter-relationship between these two systems is essential for the formation of properly shaped and sealed autophagosomes. Interestingly, alteration of either complex results in the formation of visibly aberrant IMs, and, in all cases, the formation of these IMs remains sensitive to wortmannin. Two conclusions can be drawn from these experiments, first, LC3 is required for closure of the $\mathrm{IM}$, and second the expansion of the IM is dependent on PI3P production at the site of recruitment.
Although the production of LC3-II is required for autophagy, and LC3-II is a bona fide marker for autophagosomes, recent reports have shown that the reverse is not the case. LC3-II can form and accumulate in the absence of autophagosomes, and in some cases, LC3-II levels were found to be insensitive to inhibitors of lysosomal enzymes in 'autophagic-flux' assays. ${ }^{17-20}$ In another example, phagocytosis involving activation of the Toll-like receptor signalling cascade resulted in the recruitment of LC3-II to the phagosome, in an Atg5, Atg7 and Beclin1-dependent reaction. ${ }^{21}$ The recruitment of LC3-II to the membrane did not result in the formation of double-membrane autophagosomes, but rather resulted in an enhanced fusion of the phagosomes with late endosomes. This observation suggests that the conjugation of LC3 to PE can occur on membranes that are either unrelated to IMs or to autophagosomal membranes, or on membranes that may be defective IMs unable to recruit the remaining machinery to make autophagosomes. The lack of LC3-II turnover in these cases also suggests that the Atg4b machinery is prevented from acting on LC3-II on these membranes, and/or that there is no fusion of the membranes with endosomes or lysosomes.

PI3K complex I and PI3P-effectors. In yeast, production of PI3P by the class III PI3-k complex I (consisting of Vps34, Vps15, Vps30/Atg6, Atg14) is essential for autophagy. Complex II (consisting of Vps34, Vps15, Vps30/Atg6, Vps38) produces the same lipid species, but has been shown to be required for the endosomal vacuolar protein sorting (Vps)-pathway. The mammalian homologues of Vps34 and Vps15, the shared lipid kinase and regulatory subunits, are called Vps34, and p150, respectively. Beclin1, the mammalian homologue of $\mathrm{Vps} 30$, has been the cornerstone for the advances in our understanding of the PI3K complex I in autophagy, and has been shown to be regulated by interaction with $\mathrm{Bcl} 2,{ }^{22}$ and $\mathrm{JNK} 1 .^{23}$ Importantly, however, the orthologues of Atg14 and Vps38 have only been identified recently to be Atg14/Barkor and ultraviolet irradiation resistant-associated gene (UVRAG), respectively. ${ }^{24-26}$

UVRAG, the homologue of Vps38, has a role in endocytosis ${ }^{27}$ and autophagy. ${ }^{26}$ Recent data suggest that its role in both pathways may be mediated by interaction with the class C Vps complex, providing a means to coordinate the two pathways to achieve a maximal autophagic response. ${ }^{27}$ In contrast, Itakura et al. ${ }^{24}$ did not detect a role for UVRAG in autophagy and further work is required to clarify this issue. Interestingly, UVRAG was shown to interact with Bax-interacting factor 1 (BIF-1), also known as endophilin $\mathrm{B} 1{ }^{28} \mathrm{BIF}-1$ was shown to be required for autophagy and was found on the surface of autophagosomes, colocalized with GFP-Atg5 and GFP-LC3. This exciting result suggests that recruitment of BIF-1 through UVRAG provides machinery to deform membranes, as BIF-1 has a N-BAR domain. BAR domains are modular protein domains, which bind membranes and cause them to undergo curvature. ${ }^{29}$ Interestingly, BIF-1 is also required for COPI vesicle budding through interaction with ARF-GAP, the GTPase activating protein for the small GTPase, ARF, thus providing additional support for its role in membrane deformation. Finally, BIF-1 was proposed to interact with $\mathrm{mAtg} 9{ }^{30}$ a multi-spanning transmembrane Atg protein, which cycles between the 
trans-Golgi network (TGN) and endosomes, and is present on autophagosomes after starvation. ${ }^{31}$

The mammalian homologue of $A \operatorname{tg} 14,{ }^{24}$ also called Barkor, ${ }^{25}$ plays a key role in the function of the mammalian PI3K complex. The presence of either Atg14/Barkor or UVRAG seems to be mutually exclusive. Thus, it seems that the two PI3K complexes found in mammalian cells are analogous to the yeast PI3K complexes I and II. Atg14/ Barkor, which, interestingly, is only found on autophagosome membranes after starvation, can localize to membranes independent of Vps34 and Beclin1. In addition, the levels of Atg14 influence the stability of both Beclin1 and Vps34. ${ }^{24}$ Thus, understanding how Atg14 is localized to the IMs may be the key for understanding the dynamics of PI3P production at the IMs. Another important question is whether, like UVRAG, Atg14 can recruit a BIF-1-like protein, and whether mAtg9 cycling from the TGN to endosomes and autophagosomes influences Atg14 recruitment.

In the autophagy pathway, PI3P produced by PI3K complex $\mathrm{I}$ is thought to be present on the IM as well as on elongating autophagosomes. Interestingly, in yeast, PI3P is preferentially localized to the inner autophagosomal membrane, ${ }^{32,33}$ where it recruits Atg18 along with Atg2. ${ }^{34}$ This recent data raises questions about where the PI3K complex I is localized on the autophagosomal membrane, and whether it produces the lipid only on the inner membrane, or all over the autophagosome, followed by segregation to the inner membrane. A localized production would obviate the need for redistribution, but require targeting of $\mathrm{PI} 3 \mathrm{~K}$ to the inner membrane. Atg18 associates with PI3P directly, whereas it has been proposed that Atg2 may associate with an unknown component on the $1 \mathrm{M} .{ }^{32}$ This unknown component may provide a clue as to how the distinct populations of PI3P are generated and recognized specifically by the autophagy machinery, and address the mystery of how and why a specific population of PI3P can recruit unique downstream effectors to catalyse autophagy.

So far, in yeast, the main direct effector of autophagosomal $\mathrm{PI} 3 \mathrm{P}$ is the Atg18-Atg2 complex. In mammalian cells, the orthologue of Atg18 was identified to be WD-repeat protein interacting with phosphoinositides (WIPI)-49, ${ }^{35}$ also called WIPI-1, which is a member of a family of four proteins called WIPI-1-4. ${ }^{36}$ Overexpressed WIPI-1 is localized to Rab5 and Rab9-positive membranes, ${ }^{35}$ and is recruited to GFP-LC3positive autophagosomes. ${ }^{36}$ Two Atg2 homologues exist in mammalian cells, which have not yet been studied, but which are predicted to contain an N-terminal Vps13-like domain. Interestingly, although the function of this short domain is not known, Vps13 functions in the endosome to Golgi transport in yeast. Further work is required on the WIPI family and mAtg2 to uncover their role in PI3P-dependent autophagosome formation.

Lastly, another PI3P effector has been identified called DFCP1 (double FYVE domain-containing protein 1) that localizes to endoplasmic reticulum (ER), ER-Golgi intermediate compartment (ERGIC) and Golgi membranes (Figure 3).

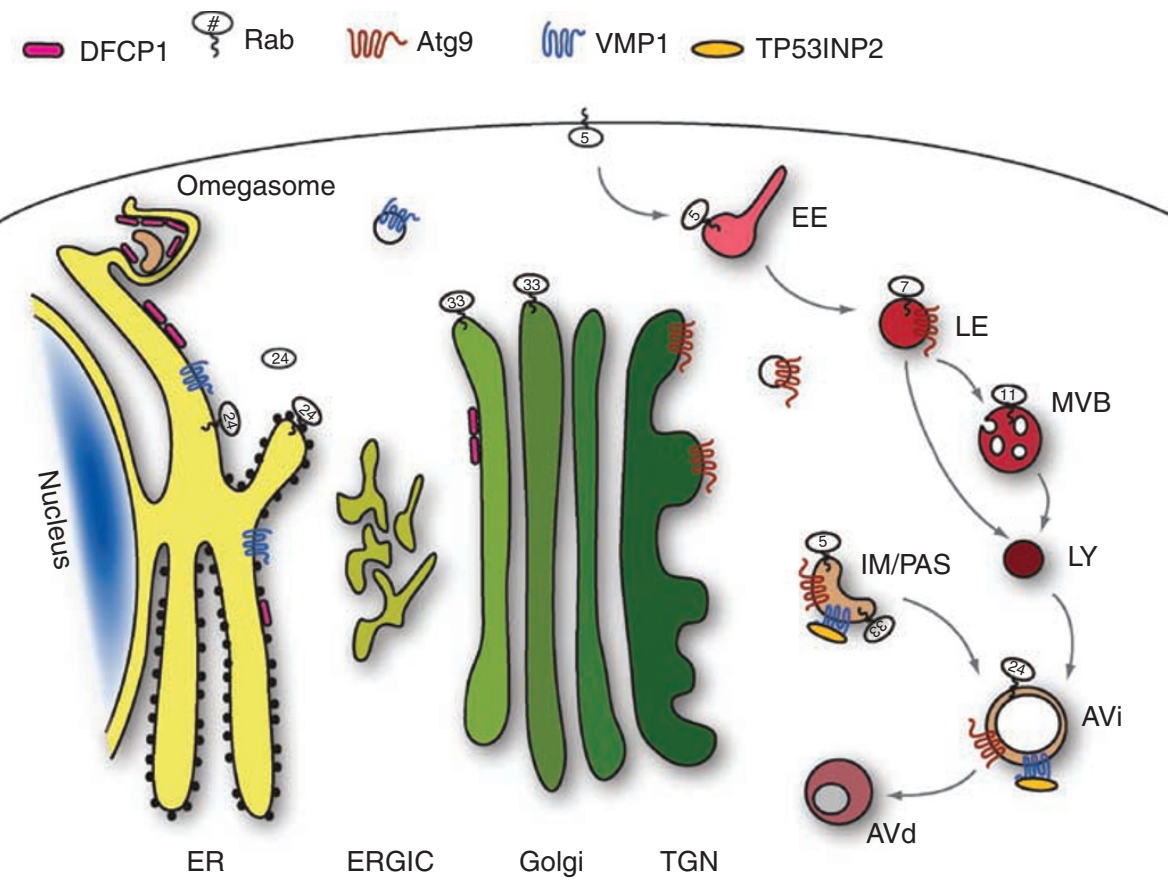

Figure 3 Intracellular localization of membrane-associated molecules involved in the formation and expansion of the isolation membrane. IM/PAS and autophagosomes are found distributed in the cytosol of mammalian cells, although there is some evidence that the maturing AVis are actively translocated to the perinuclear region. Shown is the subcellular localization of membrane proteins that are required for autophagy. Also included is TP53INP2, which associates with VMP. In many cases, the induction of autophagy causes a translocation of proteins to the IM or autophagosome from either the cytosol (LC3 and Rab proteins) or their steady-state location (mAtg9). Rab5, 7, 11, and 33 are efficiently geranylgeranylated, whereas Rab24 is inefficiently modified. ${ }^{80}$ DFCP1 is thought to remain associated with the endoplasmic reticulum (ER) by binding to $\mathrm{PI} 3 \mathrm{P}$ on the ER, and transiently interacting with the IM. For simplicity, the Atg proteins, except for Atg9, are not shown. TP53INP2, tumour protein 53-induced nuclear protein 2; VMP, vacuolar membrane protein; ERGIC; ER-Golgi intermediate compartment; TGN, trans-Golgi network; EE; early endosome, LE; late endosome, MVB; multi-vesicular body, LY; lysosome 
After amino acid starvation, DFCP1 translocates to a punctate compartment, which has been suggested to be a platform for the expansion of IMs and recruitment of Atg proteins, in particular, LC3 and Atg5.$^{37}$ It remains to be determined what role this protein has in autophagosome formation, and whether this represents a specialized form of autophagy or whether DFCP1 is essential in all autophagic processes.

ULK1 and ULK2. In yeast, the serine/threonine kinase Atg1 is known to be a key effector for the induction of autophagy, acting downstream of the target of rapamycin (TOR) protein. Atg1 functions as part of a large complex with other Atg proteins, including Atg13 and Atg17. Recent studies have shown that the mAtg1 homologues, ULK1 and 2, have regulatory roles in autophagy and also have identified ULK1/2-interacting proteins. ${ }^{19,38,39}$ Despite the high similarity between ULK1 and ULK2 (52\% overall identity) they seem to have different roles, in particular, with regard to mAtg9 trafficking. ${ }^{31}$ In addition, siRNA knockdown of ULK1 inhibits autophagy in HEK293A and HeLa cells, whereas ULK2 depletion does not. ${ }^{39}$ The basis for these differences is not known. However, recent experiments have identified three new interacting proteins, which bind both ULK1 and ULK2: the novel mammalian Atg13 homologue (KIAA0652), focal adhesion kinase family interacting protein of $200 \mathrm{kD}$ (FIP200), and Atg101 (see for review Chan and Tooze ${ }^{40}$ ).

Yeast Atg13 is a phosphoprotein that, after inactivation of TOR, is dephosphorylated, binds Atg1, and together with Atg17 regulates its activity to promote autophagy. The mAtg13 has recently been identified and was shown to be required for autophagy. ${ }^{38,41-44}$ It binds ULK1 and ULK2 at the C-terminal domain (CTD) independent of its phosphorylation state; however, mAtg13 was shown to be a substrate for ULK1 and ULK2. ${ }^{38,41-43}$ Interestingly, ULK1/2 and mAtg13 form a tight association with membranes and exhibit a partial colocalization. $^{38}$

The FIP200 also interacts with ULK1 and ULK2, ${ }^{19}$ and may be the mammalian equivalent of Atg17. ${ }^{45} \mathrm{FIP} 200$ is required for autophagy, and colocalizes with ULK1/2 and Atg16L on IMs. FIP200 also interacts with ULK1/2 in the CTD, and is required for the recruitment of ULK1/2 to IMs. In addition, it has been shown that ULK1-Atg13-FIP200 are present in a large complex that, in nutrient-rich conditions, contains mTOR. ${ }^{42}$ Atg101, identified through its homology to a Atg1binding protein in Drosophila, was shown to interact with the ULK1-Atg13-FIP200 complex in an Atg13 dependent manner. ${ }^{44}$

The identification and characterization of potential ULK $1 / 2$ effectors will allow a better understanding of the function of these kinases in autophagosome formation. The two most promising avenues would be a further understanding of the membrane association of ULK1/2, mAtg13, and mTOR, how and where the kinases are activated, and how their activity regulates the initiation of autophagy and expansion of the IM.

Transmembrane proteins. Two transmembrane proteins have so far been found to be involved in mammalian autophagy. One is the homologue of yeast Atg9, ${ }^{46}$ called mAtg9, ${ }^{31}$ or Atg9L1, ${ }^{47}$ and the other is vacuolar membrane protein 1 (VMP1). ${ }^{48}$ Although absent in yeast, there is a
VMP1 homologue in Dictyostelium discoideum, which functions in organelle biogenesis, the secretory pathway, multicellular development and, potentially, in autophagy. ${ }^{49}$ These proteins localize to different compartments in the cell, yet both contribute to the formation of autophagosomes (see Figure 3).

$\mathrm{mAtg} 9$ has six transmembrane domains with both the $\mathrm{N}$ and $\mathrm{C}$-termini in the cytosol. Although its function is not yet known, mAtg9 cycles between the TGN and late endosomes in an ULK1-dependent manner, and relocates to a peripheral pool upon starvation or rapamycin treatment. This peripheral pool was shown to overlap with both GFP-LC3-positive, Rab7-negative and GFP-LC3-positive, Rab7-positive autophagosomes, representing $\mathrm{AVis}$ and $\mathrm{AVds}$, respectively. siRNA-mediated knock down of mAtg9 resulted in an impairment of autophagy. ${ }^{31}$ From the existing data in yeast, mAtg9 has been proposed to deliver lipids to the forming autophagosome, ${ }^{50}$ an attractive hypothesis that remains to be tested experimentally in mammalian cells.

VMP1 is predicted to be a multi-spanning transmembrane protein, but unlike mAtg9, it localizes predominantly to the ER. The topology of VMP1 remains to be determined, however, the C-terminus contains a domain that interacts with Beclin1, suggesting that this domain is cytosolic. It was found that both VMP1 mRNA and protein levels increase after starvation. VMP1 has been shown to colocalize with LC3 and Beclin1 on autophagosome membranes. ${ }^{51}$ Overexpression of VMP1 induces autophagosomes even under nutrient-rich conditions and this seems to be dependent on its direct binding to Beclin1. Importantly, depletion of VMP1 by RNAi inhibits autophagosome formation after starvation or rapamycin treatment. These findings suggest a role for VMP1 in recruitment of Beclin1 along with the other components of the PI3K complex I to the IM, which leads to the recruitment of further Atg-proteins.

Recently, a novel VMP1-interacting protein, called tumour protein 53-induced nuclear protein 2 (TP53INP2), has been identified and was discovered to be essential for autophagy. ${ }^{52}$ Interestingly, TP53INP2 translocates from the nucleus to autophagosomes upon induction of autophagy, where it binds to LC3 and other LC3-family members (Gate-16, GABARAP), as well as VMP1. Therefore, TP53INP2 was proposed to act as a scaffold protein to recruit other Atg proteins to the IM. TP53INP2 is absent in ancient eukaryotes and it is tempting to speculate that it has evolved later to regulate the more diverse functions of autophagy in multicellular organisms.

It remains to be determined what the function of mAtg9 or VMP1 is; do they function as lipid carriers or provide platforms for recruitment of Atg-proteins and/or other effectors to the IM? Further study of these proteins and their binding partners should give us valuable information about autophagosome formation.

\section{Membrane Trafficking Proteins Required for Autophagosome Formation}

We propose four potential models to illustrate how an IM and autophagosome may form (see Figure 4). In developing each model, we have identified a set of common requirements, some of which are Atg proteins, and some based on data from other membrane trafficking and membrane biogenesis 
1) Lipid delivery/de novo synthesis
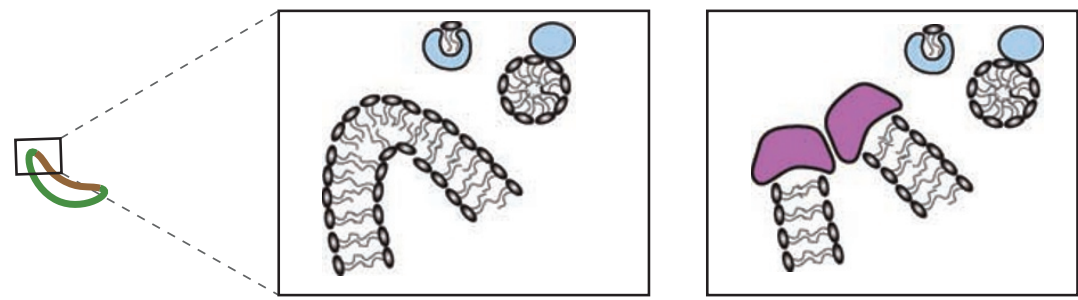

2) Vesicular transport
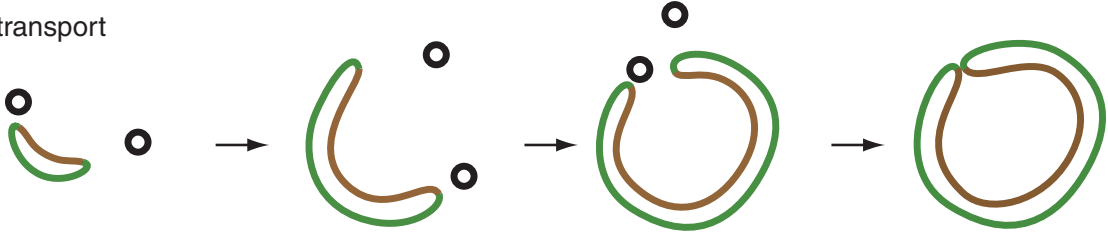

3) Cisternal assembly

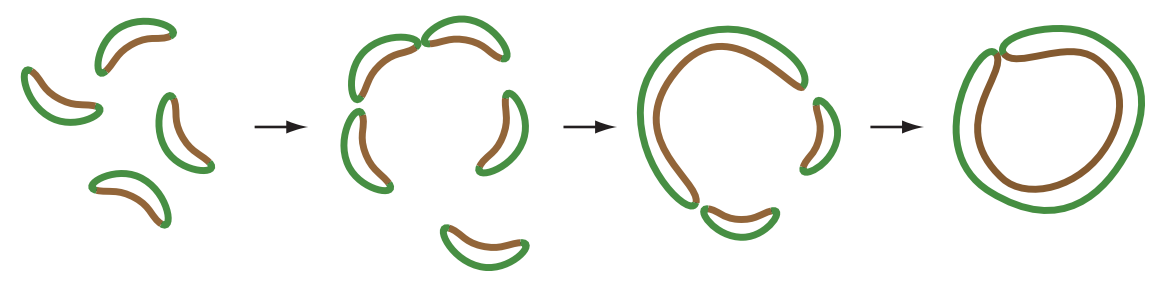

4) Membrane remodelling/extension
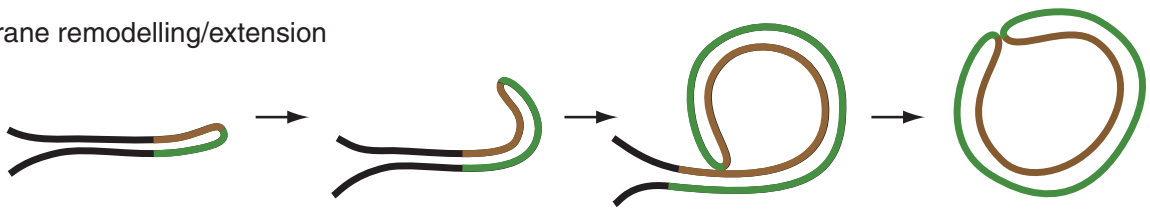

Figure 4 Four models for isolation membrane (IM) expansion. The mechanisms driving the growth and expansion of the IM are not well-understood. There are many potential scenarios by which the IM can expand, and as discussed in the text, we propose four possible alternative models. Model 1 is based on a de novo delivery of lipids by either lipid transfer proteins (light blue) to sealed bilayers (left side) or to open bilayers stabilized by a putative capping protein (purple). Models 2 and 3 both use conventional trafficking pathways through heterotypic fusion of vesicles (Model 2) or homotypic fusion (Model 3) of individual IMs. In Models 2 and 3, it is assumed that the IM is a preexisting, and perhaps stable vesicle or double-membrane structure. Model 4 proposes that the IM is derived and expands from a compartment, such as the endoplasmic reticulum. Segregation of membrane proteins and lipids into the elongating tip, and retention of resident proteins and lipids would generate a unique membrane domain. This unique domain could either bend and enclose cytosol, while still attached to the donor organelle, and then pinch off, or first pinch off as a large sheet, and then undergo curvature and closure

pathways. For brevity, we have not included a comprehensive assessment of the role of the cytoskeleton in autophagosome formation. After a discussion below of the common requirements, we will discuss these models and hypothesize on the specific cellular machinery that would be required for each model.

Curvature machinery. Regardless of the mechanism by which autophagosomes are assembled, a principal requirement is a membrane deforming and/or lipid organizing machinery to induce membrane curvature. The best characterized curvature-generating machineries are coat protein complexes (clathrin, COPI, and COPII), and proteins or protein complexes capable of bending membranes, for instance ENTH and BAR domain-containing proteins. ${ }^{29}$ So far, there are two main candidates known to be required for autophagy, which may drive IM curvature.

All Atg proteins, apart from LC3/Atg8, exhibit some preference for localization to the external surface of the nascent autophagosome. ${ }^{8}$ The Atg16 complex, in particular, has been proposed to act as a proteinaceous coat. ${ }^{53}$ Importantly, the complex dissociates from the membrane during the formation process, which is a common feature of other coat machinery. BIF-1 is a second possible candidate to fulfil this function, as it has a BAR and an $\mathrm{SH} 3$ domain, both of which are required to induce autophagosome formation through activation of the class III PI3K complex. ${ }^{28}$ Interestingly, at least in yeast, PI3P seems to be localized primarily to the inner autophagosome bilayer. ${ }^{32}$ Atg16L, BIF-1, or both together, combined with a differential distribution of lipid, could generate a lipid and protein asymmetry that could be responsible for driving membrane curvature intrinsically, or through the recruitment of additional proteins.

Expansion, closure, and fusion machinery. Another common requirement in all four models is for the machinery to expand and then close the nascent autophagosomes, allowing subsequent fusion with early and late endosomes, 
lysosomes and multi-vesicular bodies (MVBs). Expansion and closure are sequential steps that are likely to be linked either through utilization of the same machinery or through interacting machinery. Although still one of the big questions, recent data provides some suggestion on how expansion of the IM may occur, and also begins to address how the closure of the IM occurs.

Microtubule-associated protein light chain 3. As discussed above, LC3 might be directly responsible for autophagosome closure, ${ }^{6}$ possibly through its intrinsic hemifusion activity first shown in vitro. ${ }^{9}$ In yeast, Atg8 has been shown to be responsible for PAS expansion, and the amount of Atg8 present directly determined the size of the completed autophagosome. ${ }^{54}$ In contrast, in mammalian cells, IM expansion did not seem to be affected by LC3-II inactivation and further work is required to reconcile these differences.

ESCRT complex machinery. Several subunits of the endosomal sorting complex required for transport (ESCRT) are essential for autophagy. Deletion of vps28 (ESCRT-I), vps25 (ESCRT-II), vps32 (ESCRT-III), or vps4 (AAA-ATPase) results in accumulation of non-degradative autophagosomes in flies. ${ }^{55}$ Likewise, loss of mSnf7-2 or overexpression of CHMP2B ${ }^{\text {Intron5 }}$ (both subunits of ESCRT-III) in cortical neurons of mice led to a similar phenotype ${ }^{56}$ showing that a functional pool of MVBs is essential for autophagosome maturation by providing target organelles for fusion. Although the data suggests that loss of ESCRT function was a result of a fusion defect, it cannot be entirely excluded that there was also a closure defect, and expanded, but not closed, IMs accumulated. As the membrane topology of a closing autophagosome is similar to the inward budding vesicle on a late endosome (see Figure 4), we speculate that components of the ESCRT complex itself may be responsible for the final closure of autophagosomes. However, it has been shown that the deletion of ESCRT proteins in yeast does not seem to affect autophagy, ${ }^{57,58}$ thus this hypothesis remains entirely speculative.

Rabs and SNARES. The final two classes of common machinery are the Rab and soluble $N$-ethylmaleimidesensitive factor attachment protein receptors (SNARE) proteins. ${ }^{59}$ Both Rabs and SNAREs act in most, if not all, membrane trafficking pathways. Rab proteins are small molecular weight GTP-binding proteins, which act as molecular switches, mediating transport and fusion of vesicles. Relevant to this discussion are the data, which suggests that Rab proteins are crucial for developing subdomains on membranes to facilitate maturation; for example, during early to late endosome maturation, Rab5-positive domains give way to Rab7-positive domains.

Regarding autophagosome formation, the early endosomal protein Rab5 has been shown to act at early formation stages through Vps34, and regulates the conjugation of Atg12 to Atg5, possibly by promoting the recruitment of Atg12 or the conjugation machinery to the PI3P and Atg5-positive IM. ${ }^{60}$

The late endosomal Rab7 has been shown to be involved in late stages of autophagosome maturation, presumably by facilitating fusion of autophagosomes with late endosomes and lysosomes (Figure 3). Likewise, Rab11, which is present on MVBs in K562 cells may promote fusion of autophagosomes with MVBs. ${ }^{61}$ Rab24 was initially shown to partially associate with GFP-LC3-positive autophagosomes. ${ }^{62}$ Rab24 is usually localized to the ER, cis-Golgi, and ERGIC, but relocalizes to punctate structures after induction of autophagy through starvation or vinblastine treatment. However, it seems to be recruited mainly to fully formed degradative autophagosomes or autolysosomes, indicating that it is not involved in vesicular extension of the IM and its exact function remains to be elucidated.

Recent evidence suggest that Rab33 might play an important role in the targeting and recruitment of Atg16 $\mathrm{L}$ to the IM. ${ }^{63}$ It was shown that the Golgi residents (Rab33a and Rab33b) bind directly to Atg16L in a GTP-dependent manner and overexpression of GFP-Rab33b causes Atg16L recruitment to the Golgi. A constitutively active mutant of Rab33b (Rab33b-Q92L) significantly increased LC3-II levels regardless of the nutrient state, but did not lead to formation of LC3positive puncta, normally found under starvation conditions. This indicates that the Atg16 complex was recruited and functional, but additional factors needed for autophagosome expansion were lacking, and therefore, no large LC3 puncta could be observed. In fact, overexpression of Rab33b-Q92L in full medium led to an accumulation of the autophagy substrate, p62/SQSTM1, suggesting an inhibition of constitutive, basal autophagy. However, after starvation, p62 was efficiently degraded indicating that perhaps when enough additional autophagy-factors were activated, the process could continue. Alternatively, the pathway for constitutive autophagy could be different from the inducible autophagy pathway, and a different set of proteins could be involved in autophagosome formation in each case.

Finally, many vesicular fusion steps in the cell are catalysed by the SNARE protein family. SNARE proteins drive membrane fusion through the formation of a four-member alpha helix bundle (the SNARE complex), comprised of cognate target and vesicle SNAREs (called $t$ and vSNAREs, respectively) on opposing membranes, which is regulated by $\mathrm{N}$-ethylmaleimide-sensitive factor (NSF). Both the distribution of each of the four SNAREs comprising the complex, and complex formation is a characteristic of each membrane compartment. In Saccharomyces cerevisiae, it has been shown that Sec18 (NSF) and Vti1 (a SNARE) are required for fusion of the AV to the vacuole; ${ }^{64}$ however, no tSNARES could be detected on the PAS, suggesting that Rabs, NSF, or SNAREs are not involved in AV biogenesis. ${ }^{58}$ Although in mammalian cells no data exists about the presence of SNAREs on the IM, Vti1b knockout mice accumulate autophagosomes, showing that this SNARE (and presumably its SNARE complex) is required for maturation, but not formation of autophagosomes. ${ }^{65}$ This leaves open the question of the role of SNARE-mediated fusion events for expansion, assembly or closure during autophagosome formation.

\section{Models for Autophagosome Formation}

Model 1: lipid delivery/de novo synthesis. This model implies that the IM is elongated through the delivery of lipids, 
lipid droplets, or micelles. Lipids from lipid droplets could be delivered by spontaneous exchange and insertion, or through lipid transfer proteins. Delivery of lipids to the outer leaflet will allow it to expand. However, for expansion of the inner leaflet, lipids delivered to the outer leaflet will have to undergo 'flip-flop' (see Figure 4, model 1). This could be accomplished by a lipid flippase enzyme. ${ }^{66}$ Alternatively, we have speculated that the IM may be comprised of two distinct bilayers held in close proximity by a protein scaffold that could also act as a capping protein. This capping protein, or protein complex could receive lipids through a lipid transfer protein.

A third alternative is that, a close proximity of the IM to the lipid synthesis machinery in the ER could provide fatty acids for de novo autophagosomal membrane formation.

Recent studies by Axe et $a l^{37}$ have found that at least some autophagosomes seem to arise from ER membraneassociated cups, called omegasomes because of their shape. These omegasomes are highly enriched in PI3P and the PI3P-binding protein termed DFCP1, the marker for omegasomes, which might provide a signalling platform for autophagosome biogenesis (see Figure 3). However, additional experiments are required to determine the exact function of DFCP1 and the omegasomes, whether lipid synthesis occurs in or around these structures, or whether they might provide ER membrane itself to the autophagosome.

Yeast or mammalian screens have not revealed any protein machinery to support our first model, but the apparent scarceness of protein in the autophagosomal membranes ${ }^{67,68}$ and the failure to identify a definitive membrane source for autophagosome biogenesis suggests that this hypothesis may be worth further investigation.

Model 2: vesicular transport. The second model proposes that the autophagosomal membrane is delivered to the growing IM through vesicular transport (Figure 4, model 2). In this case, one would expect a similar set of trafficking machinery to be necessary as in other regulated vesicular transport events. This includes cytoskeletal motor proteins to ensure directional transport, tethers to bridge the vesicle to the target, Rab GTPases and their effectors, as well as SNARE proteins to mediate homotypic and heterotypic fusion events. ${ }^{59}$

Microtubules have been shown to facilitate autophagosome formation in mammalian cells, ${ }^{69,70}$ although it is not clear what role they have in $\mathrm{AV}$ formation. The motor protein required for transport of the autophagosome to the endosomal compartments has been identified as dynein; ${ }^{71}$ however, there is no evidence yet that this motor is required for IM expansion or closure.

Interestingly, there may be a link, which has not yet been investigated, between microtubules and the Atg machinery. ULK1/Atg1 is not only an important early regulator of autophagy, ${ }^{39,72,73}$ but has also been shown to be involved in neurite extension and axonal vesicular transport in the mouse and fly, respectively. ${ }^{74,75}$ The Drosophila homologue of ULK1/ Atg1 has recently been shown to mediate neuronal vesicular trafficking by binding and phosphorylating UNC-76, a kinesin heavy chain adaptor protein, and possibly other targets. This activated form of UNC-76 binds to synaptic vesicle proteins, and thereby links these vesicles to kinesin and the microtubule cytoskeleton for transport to the axon tip. Thus, it is tempting to speculate that ULK1 activity, which is a very early requirement in induction of autophagy, may promote transport of vesicles to the IM.

Lastly, of the Rab proteins listed above, Rab5 and Rab33b seem to be the most promising candidates. Rab proteins are recruited to specific membranes by a network of Rab effector proteins that form a membrane-specific platform. ${ }^{76}$ No autophagy-specific Rab effectors have been identified, and thus it is not yet clear how these Rab proteins would be recruited to the $\mathrm{IM}$.

Model 3: cisternal assembly. This model proposes that the autophagosome is assembled by homotypic fusion of special autophagy vesicles or discrete IMs. A similar model has been proposed earlier (see also Reggiori et $a{ }^{58}$ ). These vesicles are either constantly present in the cytoplasm and come together after induction of autophagy, or they are generated in response to autophagic stimuli. In the latter possibility, of course, the questions of how and where they would arise are raised, and their creation may involve special fission machinery. Otherwise, similar components would be needed, as in the vesicular transport model, with a homotypic instead of heterotypic fusion machinery.

Model 4: membrane remodelling/extension. In this model, the autophagosome is not so much generated by gradual expansion of the IM, but by extension and curvature of a pre-existing membrane sheet, or even by flattening an endosome-like structure. An analogy for such membrane remodelling/extension is the biogenesis of peroxisomes from specialized ER subdomains, in which ER-resident proteins are excluded and peroxisomal proteins get enriched. ${ }^{77}$

The first scenario (extension and curvature) would require some kind of selection and/or exclusion machinery to determine which lipids and proteins would enter into the autophagosome membrane and which would be excluded. The second possibility (flattening) would require a protein scaffold to deform the membrane and a mechanism to alter the lipid composition as the membrane changes shape. On the basis of immunofluorescence and EM data, it is often assumed that the IM is a crescent shaped membrane vesicle, distributed randomly throughout the cytoplasm. ${ }^{8}$ In this scenario, however, the IM would be more like a signalling platform attached to the donor membrane structure. Seen in cross-section by EM, the sorted domain with its selective localization of proteins would resemble a cup-shaped structure.

\section{Conclusions}

Our first three hypothetical models are based on pre-existing, largely established mechanisms for lipid movement between membranes, and vesicular trafficking pathways. The last model is based on speculation, but was developed from the notion that the IM arises from the ER. ${ }^{78}$ It should be noted that these models could coexist and drive different stages of autophagy. For example, biogenesis of the PAS/IM might use one mechanism, whereas expansion would employ another. 
Further advances in our understanding could come from the identification of early intermediates, either visualized by sophisticated microscopy techniques, or using conventional approaches after manipulation of the process to accumulate intermediates. In addition, the continued identification of novel effectors of the key players will allow a greater understanding of the function of the proteins localized to the IM.

Acknowledgements. We thank members of the laboratory for helpful discussions, and Drs. Fiona McAlpine, Andrea Orsi, Ed Chan, and John Tooze for reading the manuscript and for their helpful comments. We thank Cancer Research UK for funding the research in the Tooze laboratory.

1. Mizushima N, Levine B, Cuervo AM, Klionsky DJ. Autophagy fights disease through cellular self-digestion. Nature 2008; 451: 1069-1075.

2. Suzuki K, Kirisako $T$, Kamada $Y$, Mizushima N, Noda T, Ohsumi $Y$. The pre-autophagosomal structure organized by concerted functions of APG genes is essential for autophagosome formation. EMBOJ 2001; 20: 5971-5981.

3. Klionsky DJ, Cregg JM, Dunn WA, Emr SD, Sakai Y, Sandoval IV et al. A unified nomenclature for yeast autophagy-related genes. Dev Cell 2003; 5: 539-545.

4. Suzuki K, Kubota $Y$, Sekito T, Ohsumi Y. Hierarchy of Atg proteins in pre-autophagosomal structure organization. Genes Cells 2007; 12: 209-218.

5. Mizushima N, Kuma A, Kobayashi Y, Yamamoto A, Matsubae M, Takao T et al. Mouse Apg16L, a novel WD-repeat protein, targets to the autophagic isolation membrane with the Apg12-Apg5 conjugate. J Cell Sci 2003; 116: 1679-1688.

6. Fujita N, Itoh T, Omori H, Fukuda M, Noda T, Yoshimori T. The Atg16L complex specifies the site of LC3 lipidation for membrane biogenesis in autophagy. Mol Biol Cell 2008; 19 2092-2100.

7. Hanada T, Noda NN, Satomi Y, Ichimura Y, Fujioka Y, Takao T et al. The Atg12-Atg5 conjugate has a novel E3-like activity for protein lipidation in autophagy. J Biol Chem 2007; 282: 37298-37302

8. Mizushima N. Dissection of autophagosome formation using Apg5-deficient mouse embryonic stem cells. J Cell Biol 2001; 152: 657-667.

9. Nakatogawa $\mathrm{H}$, Ichimura $\mathrm{Y}$, Ohsumi $\mathrm{Y}$. Atg8, a ubiquitin-like protein required for autophagosome formation, mediates membrane tethering and hemifusion. Cell 2007; 130: $165-178$.

10. Kimura $\mathrm{S}, \mathrm{Noda} T$, Yoshimori $T$. Dissection of the autophagosome maturation process by a novel reporter protein, tandem fluorescent-tagged LC3. Autophagy 2007; 3: 452-460.

11. Marino G, Uria JA, Puente XS, Quesada V, Bordallo J, Lopez-Otin C. Human autophagins, a family of cysteine proteinases potentially implicated in cell degradation by autophagy. J Biol Chem 2003; 278: 3671-3678.

12. Hemelaar J, Lelyveld VS, Kessler BM, Ploegh HL. A single protease, Apg4B, is specific for the autophagy-related ubiquitin-like proteins GATE-16, MAP1-LC3, GABARAP and Apg8L. J Biol Chem 2003; 278: 51841-51850.

13. Kabeya Y, Mizushima N, Yamamoto A, Oshitani-Okamoto S, Ohsumi Y, Yoshimori T. LC3, GABARAP and GATE16 localize to autophagosomal membrane depending on form-II formation. J Cell Sci 2004; 117: 2805-2812.

14. Tanida I, Sou Y-S, Ezaki J, Minematsu-keguchi N, Ueno T, Kominami E. HsAtg4B/ HsApg4B/autophagin-1 cleaves the carboxyl termini of three human Atg8 homologues and delipidates microtubule-associated protein light Chain 3- and GABAA receptor-associated protein-phospholipid conjugates. J Biol Chem 2004; 279: 36268-36276.

15. Fujita N, Hayashi-Nishino M, Fukumoto H, Omori H, Yamamoto A, Noda T et al. An Atg4B mutant hampers the lipidation of LC3 paralogues and causes defects in autophagosome closure. Mol Biol Cell 2008; 19: 4651-4659.

16. Sou Y-S, Waguri S, Iwata J-I, Ueno T, Fujimura T, Hara T et al. The Atg8 conjugation system is indispensable for proper development of autophagic isolation membranes in mice. Mol Biol Cell 2008; 19: 4762-4775.

17. Zeng $\mathrm{X}$, Overmeyer $\mathrm{JH}$, Maltese WA. Functional specificity of the mammalian Beclin-Vps34 PI 3-kinase complex in macroautophagy versus endocytosis and lysosomal enzyme trafficking. J Cell Sci 2006; 119: 259-270.

18. Matsui $\mathrm{Y}$, Takagi $\mathrm{H}, \mathrm{Qu} \mathrm{X}$, Abdellatif M, Sakoda $\mathrm{H}$, Asano $\mathrm{T}$ et al. Distinct roles of autophagy in the heart during ischemia and reperfusion: roles of AMP-activated protein kinase and Beclin 1 in mediating autophagy. Circ Res 2007; 100: 914-922.

19. Hara T, Takamura A, Kishi C, lemura S, Natsume T, Guan JL et al. FIP200, a ULKinteracting protein, is required for autophagosome formation in mammalian cells. J Cell Biol 2008; 181: 497-510.

20. Ueno T, Wataru Sato W, Yasuo Horie Y, Masaaki Komatsu M, Isei Tanida I, Mitsutaka Yoshida $\mathrm{M}$ et al. Loss of Pten, a tumor suppressor, causes the strong inhibition of autophagy without affecting LC3 lipidation. Autophagy 2008; 4: 692-700.

21. Sanjuan MA, Dillon CP, Tait SWG, Moshiach S, Dorsey F, Connell S et al. Toll-like receptor signalling in macrophages links the autophagy pathway to phagocytosis. Nature 2007; 450 : 1253-1257.
22. Pattingre S, Tassa A, Qu X, Garuti R, Liang XH, Mizushima N et al. Bcl-2 antiapoptotic proteins inhibit beclin 1-dependent autophagy. Cell 2005; 122: 927-939.

23. Wei Y, Pattingre S, Sinha S, Bassik M, Levine B. JNK1-mediated phosphorylation of Bcl-2 regulates starvation-induced autophagy. Mol Cell 2008; 30: 678-688.

24. Itakura $\mathrm{E}$, Kishi $\mathrm{C}$, Inoue $\mathrm{K}$, Mizushima N. Beclin 1 forms two distinct phosphatidylinosito 3-kinase complexes with mammalian Atg14 and UVRAG. Mol Biol Cell 2008; 19: 5360-5372.

25. Sun Q, Fan W, Chen K, Ding X, Chen S, Zhong Q. Identification of Barkor as a mammalian autophagy-specific factor for Beclin 1 and class III phosphatidylinositol 3-kinase. Proc Nat Acad Sci USA 2008; 105: 19211-19216.

26. Liang C, Feng P, Ku B, Dotan I, Canaani D, Oh B-H et al. Autophagic and tumour suppressor activity of a novel Beclin1-binding protein UVRAG. Nat Cell Biol 2006; 8 688-698.

27. Liang C, Lee J-S, Inn K-S, Gack MU, Li Q, Roberts EA et al. Beclin1-binding UVRAG targets the class $C$ Vps complex to coordinate autophagosome maturation and endocytic trafficking. Nat Cell Biol 2008; 10: 776-787.

28. Takahashi Y, Coppola D, Matsushita N, Cualing HD, Sun M, Sato Y et al. Bif-1 interacts with Beclin 1 through UVRAG and regulates autophagy and tumorigenesis. Nat Cell Biol 2007; 9: 1142-1151.

29. Itoh T, De Camilli P. BAR, F-BAR (EFC) and ENTH/ANTH domains in the regulation of membrane-cytosol interfaces and membrane curvature. Biochimica et Biophysica Acta (BBA) - Mol Cell Biol Lipids 2006; 1761: 897-912.

30. Takahashi $Y$, Meyerkord CL, Wang HG. BARgaining membranes for autophagosome formation: regulation of autophagy and tumorigenesis by Bif- $1 /$ Endophilin B1. Autophagy 2008; 4: 121-124.

31. Young ARJ, Chan EYW, Hu XW, chl R, Crawshaw SG, High S et al. Starvation and ULK1dependent cycling of mammalian Atg9 between the TGN and endosomes. J Cell Sci2006; 119: 3888-3900.

32. Obara K, Ohsumi Y. Dynamics and function of Ptdlns(3)P in autophagy. Autophagy 2008; 4: 952-954

33. Obara K, Noda T, Niimi K, Ohsumi Y. Transport of phosphatidylinositol 3-phosphate into the vacuole via autophagic membranes in Saccharomyces cerevisiae. Genes Cells 2008; 13: 537-547.

34. Obara K, Sekito T, Niimi K, Ohsumi Y. The Atg18-Atg2 complex is recruited to autophagic membranes via phosphatidylinositol 3-phosphate and exerts an essential function. J Biol Chem 2008; 283: 23972-23980.

35. Jeffries TR, Dove SK, Michell RH, Parker PJ. Ptdlns-specific MPR pathway association of a novel WD40 repeat protein, WIPI49. Mol Biol Cell 2004; 15: 2652-2663.

36. Proikas-Cezanne T, Waddell S, Gaugel A, Frickey T, Lupas A, Nordheim A. WIPI-1 (WIPI49), a member of the novel 7-bladed WIPI protein family, is aberrantly expressed in human cancer and is linked to starvation-induced autophagy. Oncogene 2004; 58 9314-9325.

37. Axe EL, Walker SA, Manifava M, Chandra P, Roderick HL, Habermann A et al. Autophagosome formation from membrane compartments enriched in phosphatidylinositol 3-phosphate and dynamically connected to the endoplasmic reticulum. J Cell Biol 2008; 182: 685-701.

38. Chan EY, Longatti A, McKnight NC, Tooze SA. Kinase-inactivated ULK proteins inhibit autophagy via their conserved C-terminal domain using an Atg13-independent mechanism. Mol Cell Biol 2009; 29: 157-171.

39. Chan EY, Kir S, Tooze SA. siRNA screening of the kinome identifies ULK1 as a multidomain modulator of autophagy. J Biol Chem 2007; 282: 25464-25474.

40. Chan EY, Tooze SA. Evolution and expansion in Atg1 function. Autophagy 2009 (in press).

41. Chang Y-Y, Neufeld TP. An Atg1/Atg13 complex with multiple roles in TOR-mediated autophagy regulation. Mol Biol Cell 2009, E08-12-1250 (in press).

42. Hosokawa N, Hara T, Kaizuka T, Kishi C, Takamura A, Miura Y et al. Nutrient-dependent mTORC1 association with the ULK1-Atg13-FIP200 complex required for autophagy. Mol Biol Cell 2009, E08-12-1248 (in press).

43. Jung CH, Jun CB, Ro S-H, Kim Y-M, Otto NM, Cao J et al. ULK-Atg13-FIP200 complexes mediate mTOR signaling to the autophagy machinery. Mol Biol Cell 2009, E08-12-1249 (in press).

44. Mercer CA, Kaliappan A, Dennis PB. A novel, human Atg13 binding protein, Atg101, interacts with ULK1 and is essential for macroautophagy. Autophagy 2009 (in press).

45. Hara T, Mizushima N. Role of ULK-FIP200 complex in mammalian autophagy: FIP200, a counterpart of yeast Atg17? Autophagy 2009; 5: 85-87.

46. Lang T, Reiche S, Straub M, Bredschneider M, Thumm M. Autophagy and the cvt pathway both depend on AUT9. J Bacteriol 2000; 182: 2125-2133.

47. Yamada T, Carson AR, Caniggia I, Umebayashi K, Yoshimori T, Nakabayashi K et al. Endothelial nitric-oxide synthase antisense (NOS3AS) gene encodes an autophagy-related protein (APG9-like2) highly expressed in trophoblast. J Biol Chem 2005; 280: 18283-18290.

48. Dusetti NJ, Jiang Y, Vaccaro Ml, Tomasini R, Azizi Samir A, Calvo EL et al. Cloning and expression of the rat vacuole membrane protein 1 (VMP1), a new gene activated in pancreas with acute pancreatitis, which promotes vacuole formation. Biochem Biophys Res Commun 2002; 290: 641-649.

49. Calvo-Garrido J, Carilla-Latorre S, Lazaro-Dieguez F, Egea G, Escalante R. Vacuole membrane protein 1 is an endoplasmic reticulum protein required for organelle biogenesis, protein secretion, and development. Mol Biol Cell 2008; 19: 3442-3453.

50. Kim J, Huang WP, Stromhaug PE, Klionsky DJ. Convergence of multiple autophagy and cytoplasm to vacuole targeting components to a perivacuolar membrane compartment prior to de novo vesicle formation. J Biol Chem 2002; 277: 763-773. 
51. Ropolo A, Grasso D, Pardo R, Sacchetti ML, Archange C, Lo Re A et al. The pancreatitisinduced vacuole membrane protein 1 triggers autophagy in mammalian cells. J Biol Chem 2007; 282: 37124-37133.

52. Nowak J, Archange C, Tardivel-Lacombe J, Pontarotti P, Pebusque MJ, Vaccaro Ml et al. The TP53INP2 protein is required for autophagy in mammalian cells. Mol Biol Cell 2009 20: 870-881.

53. Reggiori F, Klionsky D. Autophagosomes: biogenesis from scratch? Curr Opin Cell Bio 2005; 17: 1-8.

54. Xie Z, Nair U, Klionsky DJ. Atg8 controls phagophore expansion during autophagosome formation. Mol Biol Cell 2008; 19: 3290-3298.

55. Rusten TE, Vaccari T, Lindmo K, Rodahl LM, Nezis IP, Sem-Jacobsen $C$ et al. ESCRTs and Fab1 regulate distinct steps of autophagy. Curr Biol 2007; 17: 1817-1825.

56. Lee J, Beigneux A, Ahmad S, Young S, Gao F. ESCRT-III dysfunction causes autophagosome accumulation and neurodegeneration. Curr Biol 2007; 17: 1561-1567.

57. Epple UD, Eskelinen EL, Thumm M. Intravacuolar membrane lysis in Saccharomyces cerevisiae. Does vacuolar targeting of Cvt17/Aut5p affect its function? J Biol Chem 2003 278: $7810-7821$.

58. Reggiori F, Wang CW, Nair U, Shintani T, Abeliovich H, Klionsky DJ. Early stages of the secretory pathway, but not endosomes, are required for Cvt vesicle and autophagosome assembly in Saccharomyces cerevisiae. Mol Biol Cell 2004; 15: 2189-2204.

59. Cai H, Reinisch K, Ferro-Novick S. Coats, tethers, Rabs, and SNAREs work together to mediate the intracellular destination of a transport vesicle. Dev Cell 2007; 12: 671-682.

60. Ravikumar B, Imarisio S, Sarkar S, O'Kane CJ, Rubinsztein DC. Rab5 modulates aggregation and toxicity of mutant huntingtin through macroautophagy in cell and fly models of Huntington disease. J Cell Sci 2008; 121: 1649-1660.

61. Fader CM, Sanchez D, Furlan M, Colombo MI. Induction of autophagy promotes fusion of multivesicular bodies with autophagic vacuoles in k562 cells. Traffic 2008; 9: 230-250.

62. Munafo DB, Colombo Ml. Induction of autophagy causes dramatic changes in the subcellular distribution of GFP-Rab24. Traffic 2002; 3: 472-482.

63. Itoh T, Fujita N, Kanno E, Yamamoto A, Yoshimori T, Fukuda M. Golgi-resident smal GTPase Rab33B interacts with Atg16 L and modulates autophagosome formation. Mol Bio Cell 2008; 19: 2916-2925 E07-12-1231.

64. Ishihara N, Hamasaki M, Yokota S, Suzuki K, Kamada Y, Kihara A et al. Autophagosome requires specific early Sec proteins for its formation and NSF/SNARE for vacuolar fusion. Mol Biol Cell 2001; 12: 3690-3702.

65. Atlashkin V, Kreykenbohm V, Eskelinen E-L, Wenzel D, Fayyazi A, Fischer von Mollard G. Deletion of the SNARE vtitb in mice results in the loss of a single SNARE partner, syntaxin 8. Mol Cell Biol 2003; 23: 5198-5207.
66. Lenoir $\mathrm{G}$, Williamson $\mathrm{P}$, Holthuis $\mathrm{J}$. On the origin of lipid asymmetry: the flip side of ion transport. Curr Opin Chem Biol 2007; 11: 654-661.

67. Baba M, Osumi M, Ohsumi Y. Analysis of the membrane structures involved in autophagy in yeast by freeze-replica method. Cell Struct Funct 1995; 20: 465-471.

68. Hirsimaki Y, Hirsimaki P, Lounatmaa K. Vinblastine-induced autophagic vacuoles in mouse liver and Ehrlich ascites tumor cells as assessed by freeze-fracture electron microscopy. Eur J Cell Biol 1982; 27: 298-301.

69. Köchl R, Hu X, Chan E, Tooze SA. Microtubules facilitate autophagosomal formation and fusion of autophagosomes with endosomes. Traffic 2006; 7: 129-145.

70. Fass E, Shvets E, Degani I, Hirschberg K, Elazar Z. Microtubules support production of starvation-induced autophagosomes but not their targeting and fusion with lysosomes. J Biol Chem 2006; 281: 36303-36316.

71. Kimura S, Noda T, Yoshimori T. Dynein-dependent movement of autophagosomes mediates efficient encounters with lysosomes. Cell Struct Funct 2008; 33: 109-122.

72. Kamada Y, Funakoshi T, Shintani T, Nagano K, Ohsumi M, Ohsumi Y. Tor-mediated induction of autophagy via an Apg1 protein kinase complex. J Cell Biol 2000; 150: 1507-1513.

73. Scott RC, Juhasz G, Neufeld TP. Direct induction of autophagy by Atg1 inhibits cell growth and induces apoptotic cell death. Curr Biol 2007; 17: 1-11.

74. Tomoda T, Kim JH, Zhan C, Hatten ME. Role of Unc51.1 and its binding partners in CNS axon outgrowth. Genes Dev 2004; 18: 541-558.

75. Toda H, Mochizuki H, Flores III R, Josowitz R, Krasieva TB, Lamorte VJ et al. UNC-51/ ATG1 kinase regulates axonal transport by mediating motor-cargo assembly. Genes Dev 2008; 22: 3292-3307.

76. Grosshans BL, Ortiz D, Novick P. Rabs and their effectors: achieving specificity in membrane traffic. Proc Natl Acad Sci USA 2006; 103: 11821-11827.

77. Geuze HJ, Murk JL, Stroobants AK, Griffith JM, Kleijmeer MJ, Koster AJ et al. Involvement of the endoplasmic reticulum in peroxisome formation. Mol Biol Cell 2003; 14: 2900-2907.

78. Dunn Jr WA. Studies on the mechanisms of autophagy: formation of the autophagic vacuole. J Cell Biol 1990; 110: 1923-1933.

79. Panaretou C, Domin J, Cockcroft S, Waterfield MD. Characterization of $\mathrm{p} 150$, an adaptor protein for the human phosphatidylinositol (Ptdlns) 3-kinase. Substrate presentation by phosphatidylinositol transfer protein to the p150 Ptdlns 3-kinase complex. J Biol Chem 1997: 272: 2477-2485.

80. Erdman RA, Shellenberger KE, Overmeyer JH, Maltese WA. Rab24 is an atypical member of the Rab GTPase family. Deficient GTPase activity, GDP dissociation inhibitor interaction, and prenylation of Rab24 expressed in cultured cells. J Biol Chem 2000; 275 : 3848-3856. 